\title{
Machine-Learned Energy Functionals for Strongly Correlated Systems
}

\author{
Daniel S. King, ${ }^{\dagger}$ Donald G. Truhlar, ${ }^{*, \ddagger}$ and Laura Gagliardi*, \\ $\dagger$ Department of Chemistry, University of Chicago, Chicago IL \\ $\ddagger$ Department of Chemistry, University of Minnesota, Minneapolis MN \\ IDepartment of Chemistry, Pritzker School of Molecular Engineering, James Franck \\ Institute, Chicago Center for Theoretical Chemistry, University of Chicago, Chicago IL \\ E-mail: truhlar@umn.edu; Igagliardi@uchicago.edu
}

\begin{abstract}
We introduce multiconfiguration data-driven functional methods (MC-DDFMs), a group of methods which aim to correct the total or classical energy of a qualitatively accurate multiconfigurational wave function using a machine-learned functional of some featurization of the wave function, such as the density or on-top density. On a dataset of carbene singlet-triplet energy splittings, we demonstrate that MC-DDFMs are able to achieve near-benchmark performance on systems not used for training with a robust degree of active space independence. This data-driven approach holds particular promise for the development of new functionals for multiconfigurational pair-density functional theory (MC-PDFT), because corrections to the CASSCF classical energy appear to be more transferable to types of molecules not included in the training data than corrections to total energies yielded by wave function methods such as CASSCF or NEVPT2.
\end{abstract}




\section{Introduction}

Although current Kohn-Sham density functional theory (KS-DFT) is highly accurate for many interesting chemical systems, it is well-known to be less accurate for strongly correlated systems than for systems well-described by a single Slater determinant. ${ }^{1-7}$ This has motivated interest in combining density functionals with multiconfigurational wave function methods $^{8-11}$ (e.g., CASSCF) that explicitly express the wave function as a superposition of electronic configurations. However, because multiconfigurational wave function methods are generally limited to a set of configurations that is too small to yield quantitatively accurate correlation energies, one must augment them by a post-MCSCF procedure in order to obtain quantitative accuracy. The most widely used of these methods include multireference perturbation theory (MRPT) ${ }^{11-14}$ (e.g., CASPT2 and NEVPT2) and multireference configuration interaction (MRCI), ${ }^{15,16}$ which are both very expensive.

As an alternative to MRPT and MRCI, we have proposed multiconfiguration pair-density functional theory (MC-PDFT) ${ }^{17}$ and multiconfiguration density-coherence functional theory (MC-DCFT). ${ }^{18}$ These methods share the feature that they compute an energy by combining wave function theory for the classical components (kinetic energy, electron-nuclear attraction, and classical electron-electron interactions) with a functional for the nonclassical components of the energy (exchange and correlation), and together they may be grouped as examples of multiconfigurational nonclassical functional theory (MC-NCFT). The general MC-NCFT energy expression is given by:

$$
E_{\mathrm{MC}-\mathrm{NCFT}}\left[\psi^{\mathrm{MC}}\right]=E_{\text {class }}^{\mathrm{MC}}+E_{\mathrm{nc}}\left[f\left[\psi^{\mathrm{MC}}\right]\right]
$$

where the classical energy $\mathrm{E}_{\text {class }}^{\mathrm{MC}}$ accounts for nucleus-nucleus repulsion, nucleus-electron attraction, classical electron-electron repulsion, and electron kinetic energy, and $E_{\mathrm{nc}}^{M C}$ is a nonclassical functional (NCF) dependent on a featurization $f$ of the reference wave func- 
tion $\psi^{\mathrm{MC}}$, which may be the density, on-top density, density coherence, gradients of these quantities, or any other featurization of the wave function.

Inspired by both the success of these methods and recent work that has used neural networks to develop density functionals for KS-DFT, ${ }^{19-22}$ we introduce a broader class of methods named "multiconfiguration density-driven functional methods" (MC-DDFMs) which aim to correct the classical or total energy $E_{\text {ref }}$ of a multiconfigurational wave function method through the use of a machine-learned functional $E_{\mathrm{ML}}$ :

$$
E_{\mathrm{MC}-\mathrm{DDFM}}\left[\psi^{\mathrm{MC}}\right]=E_{\mathrm{ref}}^{\mathrm{MC}}+E_{\mathrm{ML}}\left[f\left[\psi^{\mathrm{MC}}\right]\right]
$$

in which $E_{\mathrm{ML}}$ plays the generalized role of $E_{\mathrm{nc}}$. In this work we introduce four new MCDDFMs which use functionals of $\rho^{M C}$ and $\Pi^{M C}$ trained to correct four different reference energies $E_{\text {ref }}$ :

1) Data driven functional '21 (DDF21), a MC-NCFT functional trained to correct the classical energy:

$$
E_{\mathrm{DDF} 21}=E_{\mathrm{class}}^{\mathrm{MC}}+E_{\mathrm{DDF} 21}\left[\rho^{\mathrm{MC}}, \Pi^{\mathrm{MC}}\right]
$$

2) $\triangle \mathrm{tPBE}-21$, a functional trained to correct the translated PBE (tPBE) energy of MCPDFT: ${ }^{17}$

$$
E_{\Delta \mathrm{tPBE}-21}=E_{\mathrm{tPBE}}^{\mathrm{MC}}+E_{\Delta \mathrm{tPBE}-21}\left[\rho^{\mathrm{MC}}, \Pi^{\mathrm{MC}}\right]
$$

3) $\triangle$ CASSCF-21, a functional trained to correct the CASSCF energy:

$$
E_{\Delta \mathrm{CASSCF}-21}=E_{\mathrm{CASSCF}}^{\mathrm{MC}}+E_{\Delta \mathrm{tPBE}-21}\left[\rho^{\mathrm{MC}}, \Pi^{\mathrm{MC}}\right]
$$


4) $\triangle$ NEVPT2-21, a functional trained to correct the NEVPT2 energy:

$$
E_{\triangle \mathrm{NEVPT} 2-21}=E_{\mathrm{NEVPT} 2}^{\mathrm{MC}}+E_{\Delta \mathrm{NEVPT} 2-21}\left[\rho^{\mathrm{MC}}, \Pi^{\mathrm{MC}}\right]
$$

Below, we present the development of these MC-DDFMs as well as three different tests of their generalization to molecules outside of the training set: (i) test data similar to training data; (ii) test data using other active spaces; and (iii) test data using aryl and biradical systems. These results are quite encouraging, and we believe that further progress in this direction - particularly towards designing new functionals for MC-NCFT - has the potential to systematically achieve low-cost quantitative accuracy for a variety of different wave function methods.

Training Geometries. We have taken our training geometries from the QMSpin database of Schwilk et al., ${ }^{23}$ which contains carbenes optimized in the singlet state using CASSCF $(2,2) /$ ccpVDZ-F12 as well as benchmark-quality vertical singlet-triplet splittings obtained using explicitly correlated multireference configuration interaction with single and double excitations and the Davidson quadruples correction (MRCISD-F12+Q). ${ }^{24-27}$ In this work we have used a subset of these carbenes that contain only carbon and hydrogen atoms.

Network Architecture. We have taken an approach very similar to the recent work of Dick and Fernandez-Serra in their development of NeuralXC. ${ }^{19}$ Atomic feature vectors for atoms $I$ are obtained by projecting the density $\rho^{\mathrm{MC}}$ and on-top density $\Pi^{\mathrm{MC}}$ onto atom-centered basis functions $\phi_{n l m}$ via quadrature:

$$
c_{n l m}^{I, \rho}=\int_{\mathbf{r}} \phi_{n l m}^{I}(\mathbf{r}) \rho^{\mathrm{MC}}(\mathbf{r}) \quad c_{n l m}^{I, \Pi}=\int_{\mathbf{r}} \phi_{n l m}^{I}(\mathbf{r}) \Pi^{\mathrm{MC}}(\mathbf{r})
$$

and these features are then made rotationally invariant by the transformations: ${ }^{19,22}$ 


$$
d_{n l}^{I, \rho}=\sum_{m} c_{n l m}^{I, \rho 2} \quad d_{n l}^{I, \Pi}=\sum_{m} c_{n l m}^{I, \rho 2}
$$

In this work we used the 108 optimized basis functions developed by Chen et. al. for featurization on each atom; ${ }^{28}$ this results in a total of 36 rotationally invariant features for each atom $I$ and density $\zeta(\rho$ or $\Pi)$ : 12 "s" features $\left(l=0, d_{1,0}^{I, \zeta} \ldots d_{12,0}^{I, \zeta}\right), 12$ "p" features $\left(l=1, d_{2,1}^{I, \zeta} \ldots d_{13,1}^{I, \zeta}\right)$, and 12 "d" features $\left(l=2, d_{3,2}^{I, \zeta} \ldots d_{14,2}^{I, \zeta}\right)$. We then input each atomic feature vector $v_{I}=\left\{d_{n l}^{I, \rho}, d_{n l}^{I, \Pi}\right\}$ into its respective element network, $f_{\lambda_{I}}$ to obtain the total energy correction:

$$
E=\sum_{I} f_{\lambda_{I}}\left(v_{I}\right)
$$

as in the work of Behler and Parrinello. ${ }^{29}$

Networks were implemented and developed in PyTorch ${ }^{30}$ from the starting point of NeuralXC available on GitHub. ${ }^{31}$ Element networks consist of an input layer, $n_{\text {layers }}$ fully connected hidden layers each with $n_{\text {nodes }}$, and a one-node output layer, with $n_{\text {layers }}$ and $n_{\text {nodes }}$ treated as hyperparameters. The GELU activation function ${ }^{32}$ was used for all nodes. 


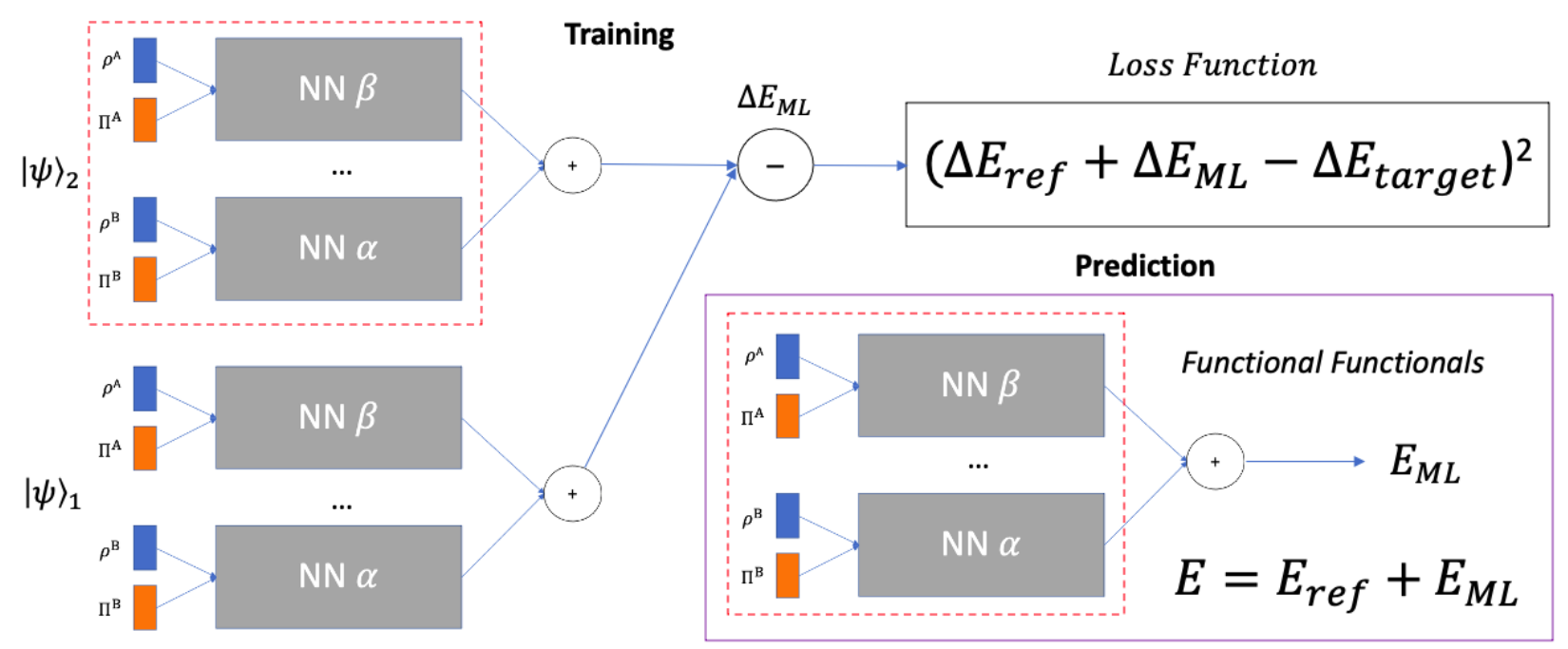

Figure 1: Network training scheme. Given a starting reference energy $E_{\text {ref }}$ with output $\Delta E_{\text {ref }}$, the element networks $\{\alpha, \beta, \ldots\}$ are regressed to minimize the mean squared deviation between corrected energy differences $\Delta E_{\mathrm{ref}}+\Delta E_{\mathrm{ML}}$ and the target energy difference $\Delta E_{\text {target }}$.

Network Training. We focus our non-classical functionals on predicting benchmark-quality energy differences between two states $|\psi\rangle_{1}$ and $|\psi\rangle_{2}$, in particular the singlet and triplet state of a single geometry. Given a difference in energy between these states from a reference method, $\Delta E_{\text {ref }}$, we train functionals to minimize the mean squared deviation between the corrected energy difference, $\Delta E_{\text {ref }}+\Delta E_{\mathrm{ML}}$, and a target energy difference, $\Delta E_{\text {target }}$ (in this work, singlet-triplet energy splittings from MRCISD-F12+Q); this training scheme is outlined in Figure 1. This centering of the loss function solely on relative energies stands in contrast to previous work in NeuralXC, ${ }^{19}$ DeepKS, ${ }^{21}$ OrbNet,${ }^{20}$ and KDFA, ${ }^{22}$ but it has three advantages: (i) it allows benchmark results to be obtained from a variety of different sources (including experiment, which almost always yields relative energies); (ii) relative energies are the quantities of most interest to chemists, since bond energies, energies of reaction, and barrier heights are all relative energies; and (iii) theoretical data used for training is almost always more accurate for relative energies than for absolute energies.

For optimization of parameters and hyperparameters, the 360 carbenes were split into a training set of 287 carbenes, a validation set of 37 carbenes, and a test set of 36 carbenes. 
All features were normalized using a StandardScaler fit on the training set, ${ }^{33}$ and networks were optimized to reduce the mean squared error loss over the entire training set in Pytorch using the Adam optimizer ${ }^{34}$ with a learning rate of 0.01 for a maximum of 20001 steps. A PyTorch scheduler (torch.optim.lr_scheduler.ReduceLROnPlateau) was used to decrease the learning rate over time upon an observed plateau in the loss to a minimum learning rate of 1.1e-7, after which the training was stopped early. The hyperparameters considered were the weight decay of the Adam optimizer and the number of nodes and layers in the element networks, and these hyperparameters were optimized using Optuna ${ }^{35}$ by minimizing loss on the validation set. The final hyperparameters of all networks and the ranges explored are given in the Supporting Information.

Wave Function Generation. State-averaged (2,2)-CASSCF wave functions, along with tPBE and NEVPT2 energies for the singlet and triplet states of each carbene, were obtained using PySCF, ${ }^{36}$ as integrated with MC-PDFT capabilities using publicly available development code. ${ }^{37}$ Atomic feature vector inputs (eq 7) were obtained via quadrature using the highest grid quality (grid_level=9). During development it was found that these input features converge at significantly lower thresholds than the CASSCF energy, and therefore more stringent CASSCF optimization parameters were used in obtaining the singlet and triplet wave functions to insure consistency (mc.conv_tol $=1 \mathrm{e}-10$, mc.conv_tol_grad $=$ 1e-6, mc.ah_lindep $=1 \mathrm{e}-14$, and mc.ah_conv_tol $=1 \mathrm{e}-12)$.

Active Space Selection. With the exception of benzene, all active spaces for CASSCF calculations were chosen automatically using the ranked-orbital approach. ${ }^{38}$ The highest 23 doubly occupied orbitals and the lowest 23 virtual orbitals of an ROHF wave function were individually Boys-localized ${ }^{39}$ and the approximate pair coefficient (APC) method $^{38}$ was employed on all doubly occupied orbitals and the localized virtual orbitals to approximate orbital entropies (the remaining virtual orbitals were not considered for the active space). These entropies were then used to rank the orbitals in terms of importance, and the final active space was selected by setting a maximum number of allowed CSFs in the wave function 
expansion (e.g., $\max (2,2), \max (4,4)$, and $\max (6,7))$ and dropping orbitals from the active space until the size of the active space satisfied the threshold. In the training data we selected all active spaces at the $\max (6,7)$ level.

Active Space Error. Although the ranked-orbital approach above is imperfect at ranking orbitals in importance for the active space, at the $\max (6,7)$ level our method failed to select active spaces with qualitatively accurate CASSCF excitation energies $(<1 \mathrm{eV}$ in absolute error) in only a small number of cases; these cases were rejected from the training, validation, and test sets. However, in addition to the calculations at the $\max (6,7)$ level that were used to train the functionals, we performed some tests with minimal active spaces generated at the $\max (2,2)$ level, which requires a perfect ranking of the orbitals; in these tests we experienced a much higher failure rate $(33 \%)$, and therefore these tests were carried out on a test subset of only 24 carbenes (listed in the Supporting Information). 


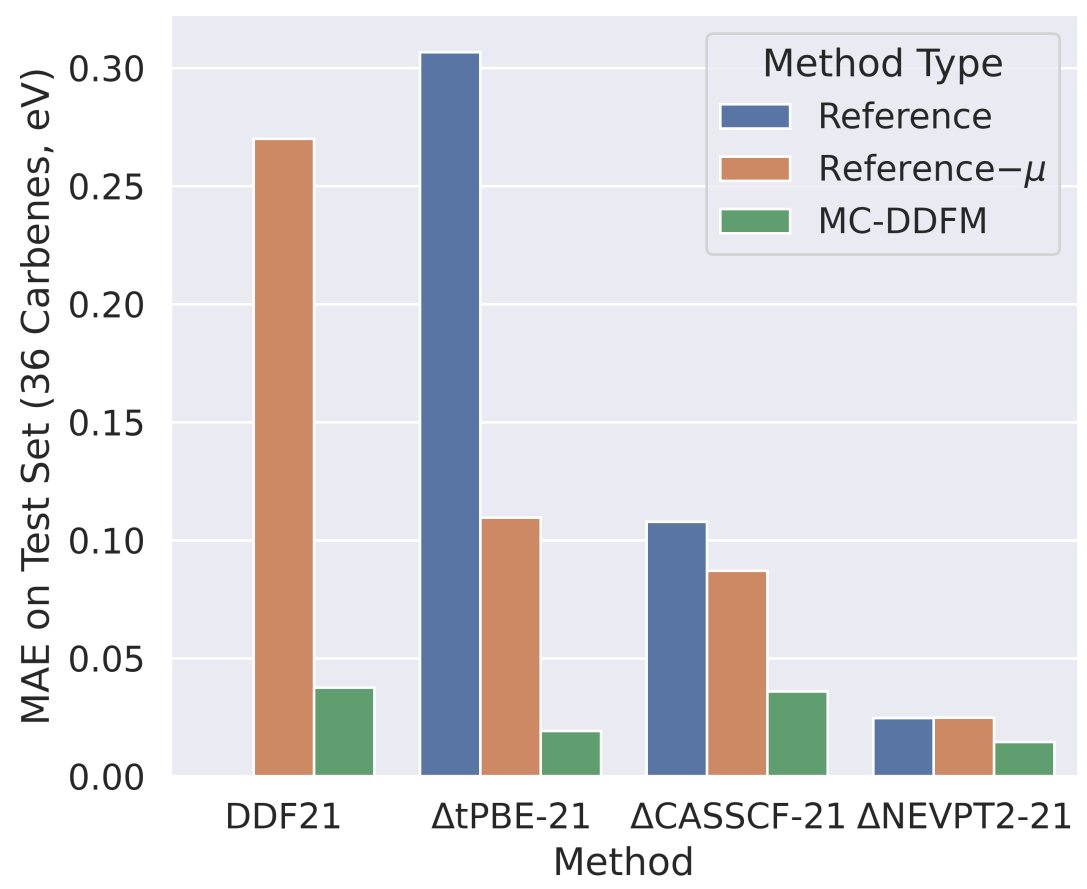

Figure 2: Mean absolute errors (MAEs) on MRCISD-F12+Q benchmark data for a test set of 36 carbenes excluded from the training data. For each MC-DDFM (DDF21, $\Delta$ tPBE-21, $\triangle$ CASSCF-21, and $\triangle$ NEVPT2-21, shown in green), we show the performance of its reference method (tPBE, CASSCF, and NEVPT2, shown in blue) as well as a one-parameter meancorrected method (Reference- $\mu$ ) shown in orange. The MAE of the CASSCF classical energy $(1.1 \mathrm{eV})$ is not shown due to scale.

Results. Figure 2 shows the performance of the four MC-DDFMs in comparison to their respective reference methods on the test set of 36 carbene singlet-triplet energy splittings. For comparison, we also show the performance of a simple one-parameter mean correction to the singlet-triplet energy splittings, in which $\Delta E_{\text {ref }}$ is corrected by its mean deviation from MRCISD-F12 $+\mathrm{Q}$ on the training data. Encouragingly, all four functionals are able to greatly improve upon these one-parameter corrections, surpassing the mean absolute errors (MAEs) of their reference methods by factors of 29 (DDF21), 16 ( $\Delta$ tPBE21), 3 ( $\Delta$ CASSCF-21), and 2 ( $\triangle$ NEVPT2-21). Additionally, although all functionals presented in the article proper depend on both the density and on-top density, additional results given in the Supporting Information show that we obtain similarly high accuracy using only density features or only 
on-top density features.

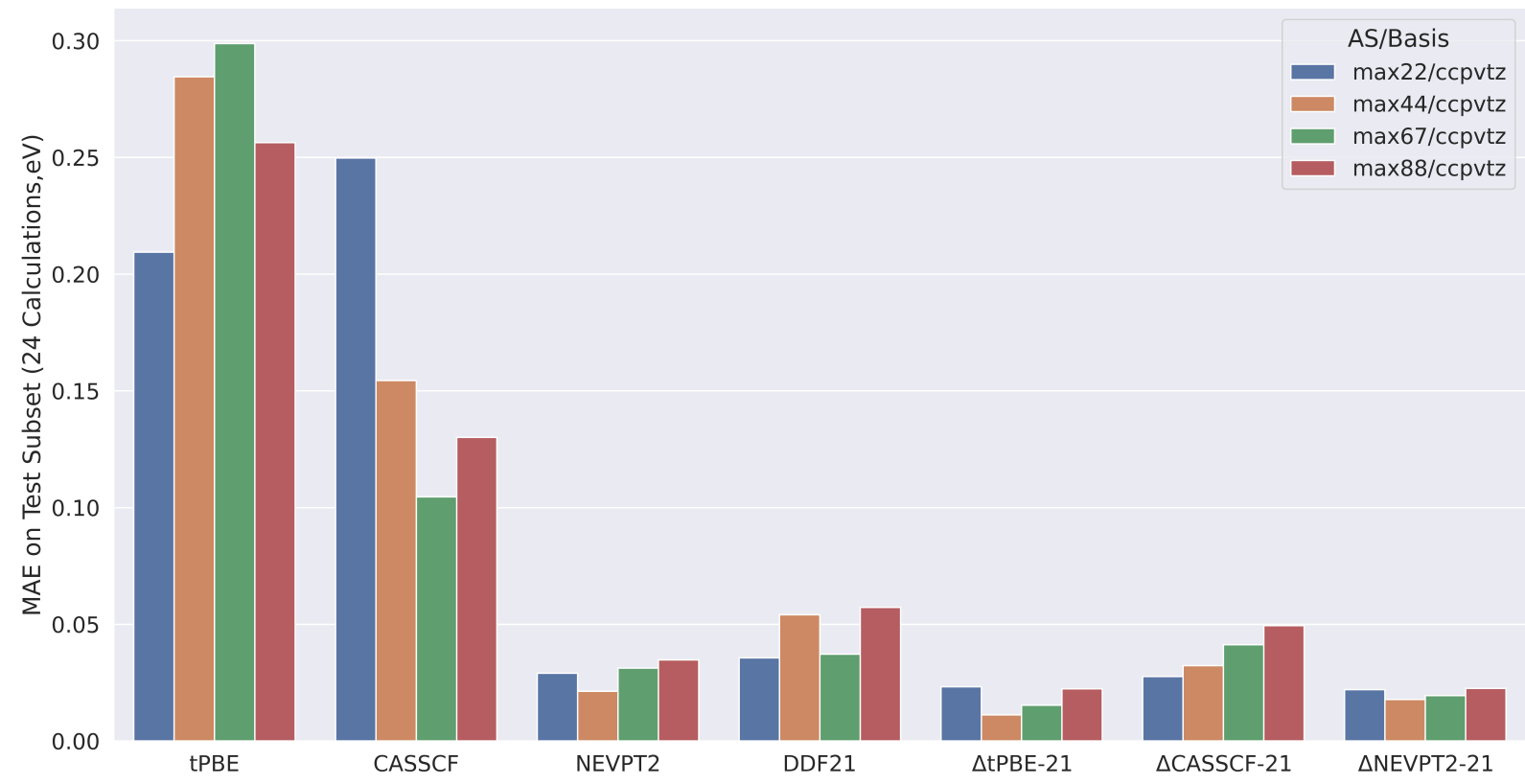

Figure 3: Mean absolute errors on MRCISD-F12 $+\mathrm{Q}$ benchmark data from a test subset of 24 carbenes for which our automated scheme chose a reasonable $(2,2)$ active space, tested with the cc-PVTZ basis at four different active space sizes: $\max (2,2), \max (4,4), \max (6,7)$, and $\max (8,8)$.

We tested the active space dependence of our data-driven functional methods on 24 carbenes with four different active space sizes whose number of configurations vary by four orders of magnitude: $\max (2,2), \max (4,4), \max (6,7)$, and $\max (8,8)$. Figure 3 shows that all MC-DDFMs maintain their near-benchmark accuracy across this wide range of active spaces, despite being trained on only $\max (6,7)$ active spaces. We note that this active space robustness is likely a result of the sole dependence of our loss function on relative energies rather than absolute ones. However, we find that one drawback of our approach is that the parameters do not seem to be easily transferable to other basis sets; when switching to either a cc-pVDZ or cc-pVQZ basis the errors of the MC-DDFMs tend to increase dramatically (Supporting Information). 


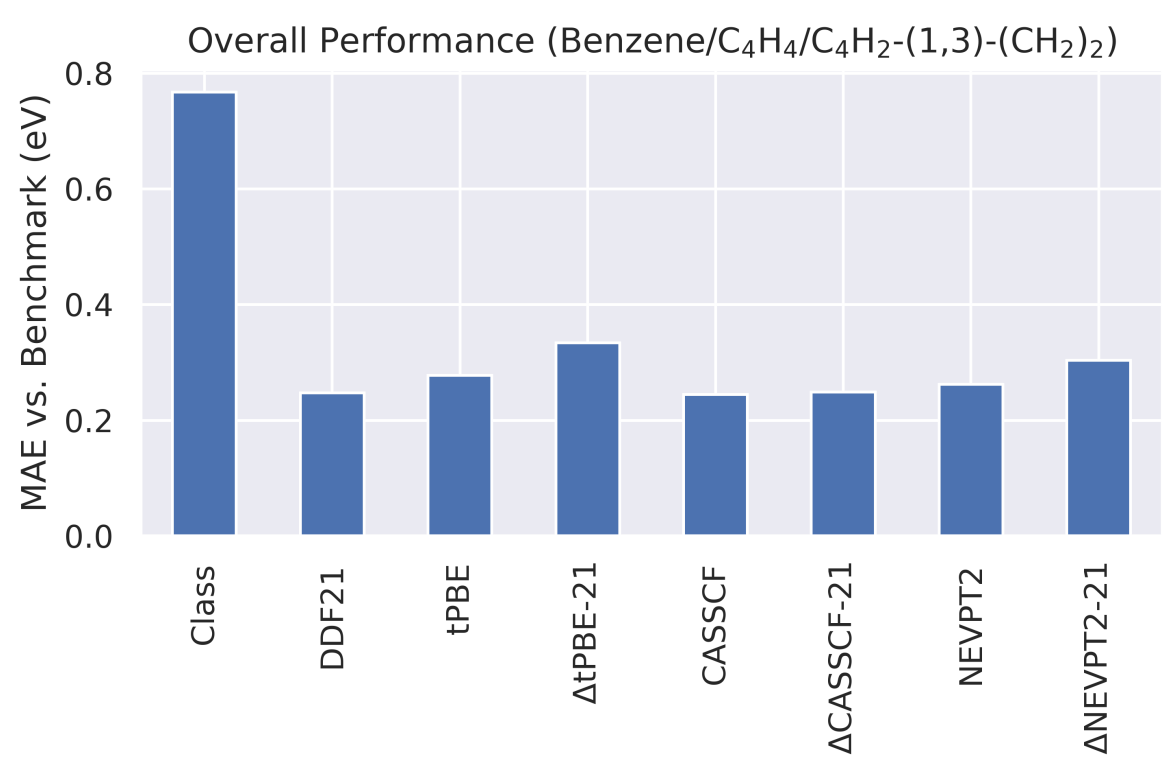

Figure 4: Mean absolute error of reference and data-driven functional methods on three difficult singlet triplet gaps, consisting of one aryl system $\left(\mathrm{C}_{6} \mathrm{H}_{6}\right.$, using the standard minimal cc-pVTZ@UNO- $(6,6)$ active space ${ }^{40}$ ) and two biradical systems (cyclobutadiene, $\mathrm{C}_{4} \mathrm{H}_{4}$, and 1,3-bis(methylene)-cyclobutadiene $\left(\mathrm{C}_{4} \mathrm{H}_{2}-(1,3)-\left(\mathrm{CH}_{2}\right)_{2}\right)$, using automatically selected $\max (10,10)$ active spaces $)$.

As a final test of generalizability, we tested the MC-DDFMs on three difficult singlet-triplet energy splittings quite different than any data in the training set: benzene and two biradical systems; cyclobutadiene $\left(\mathrm{C}_{4} \mathrm{H}_{4}\right)$ and 1,3-bis(methylene)cyclobutadiene $\left(\mathrm{C}_{4} \mathrm{H}_{2}-(1,3)-\left(\mathrm{CH}_{2}\right)_{2}\right)$ (Figure 4). These systems were taken from previous benchmark studies on translated functionals, ${ }^{41,42}$ with benchmarks for benzene taken from experiment ${ }^{43}$ and benchmarks for the biradicals from theoretical results. ${ }^{44}$ While MC-DDFMs correcting total energies $(\Delta$ tPBE21, $\triangle$ CASSCF-21, and $\triangle$ CASSCF-21) all performed worse on average than their respective reference methods, DDF21 maintains a large improvement upon the CASSCF classical energy, reducing its MAE from $0.77 \mathrm{eV}$ to only $0.25 \mathrm{eV}$. This suggests that corrections to the classical energy - as is done in MC-NCFT - may be more transferable to types of molecules not included in the training data than corrections to "complete" methods such as CASSCF or NEVPT2. Similar generalizability in this regard is achieved by MC-DDFMs trained solely on the density or on-top density (Supporting Information). 
Conclusions. We have presented a data-driven approach to the development of energy functionals for strongly correlated systems utilizing neural networks parametrized in terms of the density and on-top density. Using a dataset of carbene singlet-triplet energy splittings taken from the QMSpin database ${ }^{23}$ we find that the new multiconfiguraional data-driven functional methods (MC-DDFMs) are able to achieve benchmark-quality accuracy on carbenes not included in the training set and improve markedly on approaches using translated MC-PDFT functionals even when extended to different active spaces. Furthermore, this work shows that data-driven functionals hold particularly great promise for multiconfigurational nonclassical functional theory, as corrections to the classical energy appear to be more transferable to types of molecules not included in the training data than corrections to total energies such as yielded by CASSCF or NEVPT2. It will be interesting to see if this good performance can be maintained when the functionals are parameterized using larger and more diverse sets of training data.

\section{Acknowledgment}

This work is supported as part of the Inorganometallic Catalysis Design Center, an Energy Frontier Research Center funded by the U.S. Department of Energy, Office of Science, Basic

Energy Sciences under Award DE-SC0012702. Additionally, the authors thank the Research Computing Center (RCC) at the University of Chicago for access to computational resources.

\section{Supporting Information Available}

Names of the carbenes included in the training, validation, test, and test subsets. Histograms showing systematic errors in reference methods. Basis set dependence of MC-DDFMs. Performance and active space and basis set dependence of MC-DDFMs trained solely on density features or solely on on-top density features. Individual performances on benzene, cyclobu- 
tadiene, and 1,3-bis(methylene)-cyclobutadiene.

\section{References}

(1) Kohn, W.; Becke, A. D.; Parr, R. G. Density Functional Theory of Electronic Structure. J. Phys. Chem. 1996, 100, 12974-12980.

(2) Scuseria, G. E; Staroverov, V. N. Chapter 24 - Progress in the Development of Exchange-Correlation Functionals. In Theory and Applications of Computational Chemistry; Dykstra, C. E., Frenking, G., Kim, K. S., Scuseria, G. E., Eds.; Elsevier: Amsterdam, 2005; pp 669-724.

(3) Cohen, A. J.; Mori-Sanchez, P.; Yang, W. Insights Into Current Limitations of Density Functional Theory. Science 2008, 321, 792-794.

(4) Cramer, C. J.; Truhlar, D. G. Density Functional Theory for Transition Metals and Transition Metal Chemistry. Phys. Chem. Chem. Phys 2009, 11, 10757.

(5) Becke, A. D. Perspective: Fifty Years of Density-Functional Theory in Chemical Physics. J. Chem. Phys. 2014, 140, 18A301.

(6) Yu, H. S.; Li, S. L.; Truhlar, D. G. Perspective: Kohn-Sham Density Functional Theory Descending a Staircase. J. Chem. Phys. 2016, 145, 130901.

(7) Verma, P.; Truhlar, D. G. Status and Challenges of Density Functional Theory. Trends Chem. 2020, 2, 302-318.

(8) Nakano, H.; Nakajima, T.; Tsuneda, T.; Hirao, K. Chapter 20 - Recent Advances in Ab Initio, Density Functional Theory, and Relativistic Electronic Structure Theory. In Theory and Applications of Computational Chemistry; Dykstra, C. E., Frenking, G., Kim, K. S., Scuseria, G. E., Eds.; Elsevier: Amsterdam, 2005; pp 507-557. 
(9) Roos, B. O. Chapter 25 - Multiconfigurational Quantum Chemistry. In Theory and Applications of Computational Chemistry; Dykstra, C. E., Frenking, G., Kim, K. S., Scuseria, G. E., Eds.; Elsevier: Amsterdam, 2005; pp 725-764.

(10) Gordon, M. S.; Schmidt, M. W. Chapter 41 - Advances in Electronic Structure Theory: GAMESS a Decade Later. In Theory and Applications of Computational Chemistry; Dykstra, C. E., Frenking, G., Kim, K. S., Scuseria, G. E., Eds.; Elsevier: Amsterdam, 2005; pp 1167-1189.

(11) Gaggioli, C. A.; Stoneburner, S. J.; Cramer, C. J.; Gagliardi, L. Beyond Density Functional Theory: The Multiconfigurational Approach To Model Heterogeneous Catalysis. ACS Catal. 2019, 9, 8481-8502.

(12) Andersson, K.; Malmqvist, P. A.; Roos, B. O.; Sadlej, A. J.; Wolinski, K. Second-Order Perturbation Theory With a CASSCF Reference Function. J. Phys. Chem. 1990, 94, $5483-5488$.

(13) Hirao, K. Multireference Møller-Plesset Method. Chem. Phys. Lett. 1992, 190, 374380.

(14) Angeli, C.; Cimiraglia, R.; Evangelisti, S.; Leininger, T.; Malrieu, J.-P. Introduction of $\mathrm{n}$-Electron Valence States for Multireference Perturbation Theory. J. Chem. Phys. 2001, 114, 10252-10264.

(15) Brown, F. B.; Shavitt, I.; Shepard, R. Multireference Configuration Interaction Treatment of Potential Energy Surfaces: Symmetric Dissociation of H2O in a Double-Zeta Basis. Chem. Phys. Lett. 1984, 105, 363-369.

(16) Werner, H.; Knowles, P. J. An Efficient Internally Contracted Multiconfiguration-reference Configuration Interaction Method. J. Chem. Phys. 1988, 89, 5803-5814. 
(17) Li Manni, G.; Carlson, R. K.; Luo, S.; Ma, D.; Olsen, J.; Truhlar, D. G.; Gagliardi, L. Multiconfiguration Pair-Density Functional Theory. J. Chem. Theory Comput. 2014, 10, 3669-3680.

(18) Zhang, D.; Hermes, M. R.; Gagliardi, L.; Truhlar, D. G. Multiconfiguration DensityCoherence Functional Theory. J. Chem. Theory Comput. 2021, 17, 2775-2782.

(19) Dick, S.; Fernandez-Serra, M. Machine Learning Accurate Exchange and Correlation Functionals of the Electronic Density. Nat. Commun. 2020, 11.

(20) Qiao, Z.; Welborn, M.; Anandkumar, A.; Manby, F. R.; Miller, T. F. OrbNet: Deep Learning for Quantum Chemistry Using Symmetry-Adapted Atomic-Orbital Features. J. Chem. Phys. 2020, 153, 124111.

(21) Chen, Y.; Zhang, L.; Wang, H.; E, W. DeePKS: A Comprehensive Data-Driven Approach Toward Chemically Accurate Density Functional Theory. J. Chem. Theory Comput. 2021, 17, 170-181.

(22) Margraf, J. T.; Reuter, K. Pure Non-Local Machine-Learned Density Functional Theory for Electron Correlation. Nat. Commun. 2021, 12.

(23) Schwilk, M.; Tahchieva, D. N.; von Lilienfeld, O. A. Large Yet Bounded: Spin Gap Ranges in Carbenes. arXiv:2004.10600 [physics] 2020, Preprint.

(24) Werner, H.-J.; Knowles, P. J. An Efficient Internally Contracted MulticonfigurationReference Configuration Interaction Method. J. Chem. Phys. 1988, 89, 5803-5814.

(25) Knowles, P. J.; Werner, H.-J. An Efficient Method for the Evaluation of Coupling Coefficients in Configuration Interaction Calculations. Chem. Phys. Lett. 1988, 145, $514-522$. 
(26) Knowles, P. J.; Werner, H.-J. Internally Contracted Multiconfiguration-Reference Configuration Interaction Calculations for Excited States. Theor. Chim. Acta. 1992, 84, 95-103.

(27) Shiozaki, T.; Knizia, G.; Werner, H.-J. Explicitly Correlated Multireference Configuration Interaction: MRCI-F12. J. Chem. Phys. 2011, 134, 034113.

(28) Chen, Y.; Zhang, L.; Wang, H.; E, W. Ground State Energy Functional With Hartree-Fock Efficiency and Chemical Accuracy. J. Phys. Chem. A 2020, 124, 71557165.

(29) Behler, J.; Parrinello, M. Generalized Neural-Network Representation of HighDimensional Potential-Energy Surfaces. Phys. Rev. Lett. 2007, 98.

(30) Paszke, A. et al. PyTorch: An Imperative Style, High-Performance Deep Learning Library. In Advances in Neural Information Processing Systems 32; Wallach, H., Larochelle, H., Beygelzimer, A., d'Alché-Buc, F., Fox, E., Garnett, R., Eds.; Curran Associates, Inc., 2019; pp 8024-8035.

(31) Dick, S. Semodi/Neuralxc. https://github.com/semodi/neuralxc, (accessed 2021$04-15)$.

(32) Hendrycks, D.; Gimpel, K. Gaussian Error Linear Units (Gelus). arXiv:1606.08415 [cs.LG] 2016, Preprint.

(33) Pedregosa, F.; Varoquaux, G.; Gramfort, A.; Michel, V.; Thirion, B.; Grisel, O.; Blondel, M.; Prettenhofer, P.; Weiss, R.; Dubourg, V., et al. Scikit-Learn: Machine Learning in Python. J. Mach. Learn. Res. 2011, 12, 2825-2830.

(34) Kingma, D. P.; Ba, J. Adam: A Method for Stochastic Optimization. arXiv:1412.6980 [cs.LG] 2014, Preprint. 
(35) Akiba, T.; Sano, S.; Yanase, T.; Ohta, T.; Koyama, M. Optuna: A Next-Generation Hyperparameter Optimization Framework. Proceedings of the 25th ACM SIGKDD International Conference on Knowledge Discovery \& Data Mining. New York, NY, USA, 2019; pp 2623-2631.

(36) Sun, Q.; Berkelbach, T. C.; Blunt, N. S.; Booth, G. H.; Guo, S.; Li, Z.; Liu, J.; McClain, J. D.; Sayfutyarova, E. R.; Sharma, S.; Wouters, S.; Chan, G. K. PySCF: The Python-based Simulations of Chemistry Framework. Wiley Interdiscip. Rev. Comput. Mol. Sci. 2018, 8.

(37) MatthewRHermes/mrh. https://github.com/MatthewRHermes/mrh, (accessed 202104-15).

(38) King, D. S.; Gagliardi, L. A Ranked-Orbital Approach to Select Active Spaces for High-Throughput Multireference Computation. J. Chem. Theory Comput. 2021, 17, $2817-2831$.

(39) Foster, J. M.; Boys, S. F. Canonical Configurational Interaction Procedure. Rev. Mod. Phys. 1960, 32, 300-302.

(40) Tóth, Z.; Pulay, P. Finding Symmetry Breaking Hartree-Fock Solutions: The Case of Triplet Instability. J. Chem. Phys. 2016, 145, 164102.

(41) Sharma, P.; Bernales, V.; Truhlar, D. G.; Gagliardi, L. Valence $\pi \pi^{*}$ Excitations in Benzene Studied by Multiconfiguration Pair-Density Functional Theory. J. Phys. Chem. Lett. 2019, 10, 75-81.

(42) Stoneburner, S. J.; Truhlar, D. G.; Gagliardi, L. MC-PDFT Can Calculate Singlet-triplet Splittings of Organic Diradicals. J. Chem. Phys. 2018, 148, 064108.

(43) Doering, J. P. Low-Energy Electron-Impact Study of the First, Second, and Third Triplet States of Benzene. J. Chem. Phys. 1969, 51, 2866-2870. 
(44) Stoneburner, S. J.; Shen, J.; Ajala, A. O.; Piecuch, P.; Truhlar, D. G.; Gagliardi, L. Systematic Design of Active Spaces for Multi-Reference Calculations of Singlet-triplet Gaps of Organic Diradicals, With Benchmarks Against Doubly Electron-Attached Coupled-Cluster Data. J. Chem. Phys. 2017, 147, 164120. 


\section{Graphical TOC Entry}

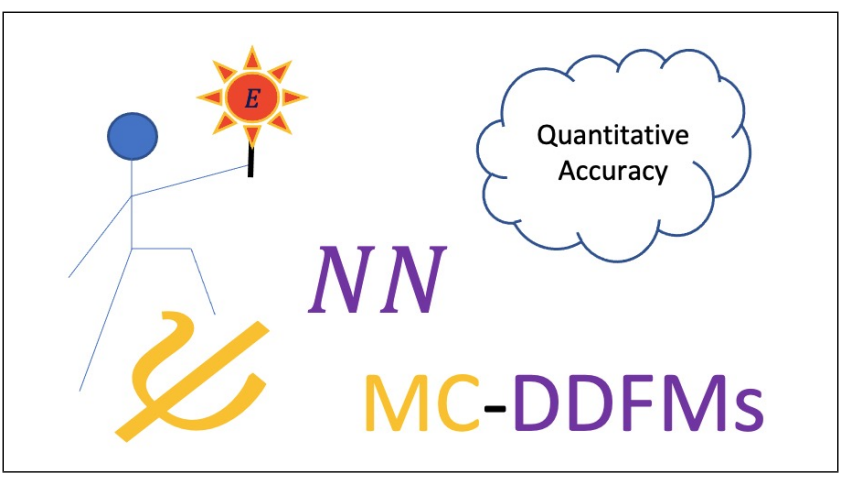

